Case Report

\title{
Use of Rotational Flap for Reconstruction of Scalp Avulsion Defect - A Case Report
}

\author{
Karthik Vishwanath ${ }^{1}$, Nikhil Shetty ${ }^{2}$, Satadru Roy ${ }^{3}$ \\ ${ }^{1,2}$ Assistant Professor, Department of Plastic Surgery, ${ }^{3}$ Post Graduate Student, Department of Oral \& M axillofacial Surgery, \\ Justice K. S. Hegde Charitable Hospital, M angalore, Karnataka, India.
}

*Corresponding Author : Satadru Roy, Post Graduate Student, Department of Oral \& Maxillofacial Surgery, A.B. Shetty Memorial Institute of Dental Sciences, Nitte University, Mangalore - 575018, Karnataka, India.

Mobile: +918584868501, E-mail: drsataroy@gmail.com

$\begin{array}{ll}\text { Received } & : 19.04 .2017 \\ \text { Review Completed } & : 05.05 .2017 \\ \text { Accepted } & : 17.05 .2017\end{array}$

Keywords : Scalp Avulsion, Scalp reconstruction, Rotation advancement flap

\begin{tabular}{|c|}
\hline Access this article online \\
\hline Quick Response Code \\
\hline
\end{tabular}

\begin{abstract}
Reconstruction of scalp defects is required for acute trauma, tumor extirpation, radiation necrosis, and the repair of traumatic alopecia or cosmetically displeasing scars. The proper choice of a reconstructive technique is affected by several factors-the size and location of the defect, the presence or absence of periosteum, the quality of surrounding scalp tissue, the presence or absence of hair, location of the hairline, and patient comorbidities.Cosmetic scalpreconstruction requires restoration and preservation of normal hair patterns and hair lines.
\end{abstract}

The scalp vertex is an area of limited scalp mobility and requires extensive undermining and recruitment of tissue from the moremobile anterior, parietal, and occipital regions. The only alternative for large defects (greater than $25 \mathrm{~cm}^{2}$ ) is large rotation-advancement flaps which require near complete scalp undermining.

This article presents a case of Acute scalp Avulsion in the Vertex and the subsequent reconstruction using a large posteriorly based Rotation-Advancement Flap.

\section{Introduction}

Scalp defects are caused by traumatisms, tumor resection surgery, radiotherapy-induced necrosis, burns and infections. ${ }^{1-3}$ The repair of such defects is dependent upon their location, size and depth. ${ }^{2}$ Unlike in other head and neck areas where local flaps are used to repair large defects, in the region of the scalp the repair of even small defects is complicated. Rotation, advancement and Trans positioning scalp flaps are the reference for reconstructing these defects. The correct design of such flaps includes preservation of the original hairline, acceptable redirectioning of the hair follicles, the incorporation of large vascular pedicles, and wound closure without excessive tension. $^{3-6}$

Knowledge of scalp anatomy is essential for preparing this flaps. ${ }^{4}$ The skin in this region is the thickest in the body. It is resistant, very scantly elastic, and is covered with hair. The subcutaneous tissue in turn contains the blood vessels, nerves and hair follicles. ${ }^{6,7}$ The main arteries irrigating the scalp are the superficial temporal artery, with its frontal and parietal branches, the posterior auricular artery and the occipital artery - all of which are branches of the external carotid artery - and the supraorbital and trochlear arteries (branches of the internal carotid artery). ${ }^{6}$ Local flaps must be based on one or two vascular pedicles of the scalp to afford a large rotation angle - thereby facilitating closure of the defect. The aponeurotic layer, also known as the epicranial aponeurosis, is the strongest layer of the scalp. As a result of its scant elasticity, the aponeurosis opposes desired flap advancement. This can be overcome by making incisions perpendicular to the direction of flap movement. ${ }^{5,6}$ correct closures with these flaps requires suturing the aponeurotic layer.

We report the case of a patient with a Traumatic Scalp 
Avulsion in the vertex region. Following wound debridement, a large defect including the pericranium remained. The defect was repaired by raising a local rotation advancementscalp flap. Following re- positioning of the flap, primary closure was done.

\section{Case Report}

A 39 year old male patient reported to the casualty of K.S. Hegde Charitable Hospital following a Road Traffic Accident. On primary assessment, a scalp avulsion defect in the vertex region of around $6 \times 4 \mathrm{~cm}$ was found, with irregular margins following the acute trauma. The percranium in the avulsed region was found to be intact. Neurosurgical Examination of the patient was performed and no intracranial haemorrhage was found on Computed Tomography. The bystanders of the patient were explained the condition of the patient and the need for him to undergo reconstruction of the avulsed portion of scalp. A written informed consent was obtained. Wound Debridement followed by posterior based Rotation Advancement local scalp flap was planned for closure of the defect.

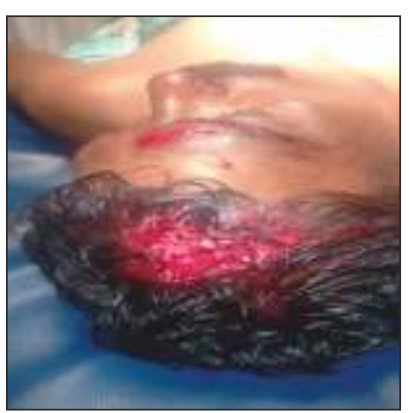

Figure 1 : Wound on presentation to casualty

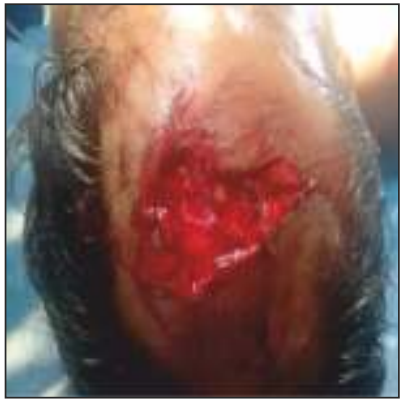

Figure 2 : Scalp Defect after head shave
Patient was put under general anesthesia. A thorough irrigation of the wound site was done to remove the lodged debris. Debridement was done following which, the unhealthy wound margins were excised and a fresh, triangular wound was created. From the posterior corner of the flap near the vertex, a curvilinear incision was placed up to the superior occipito-parietal region. Raney Scalp clips were placed along the wound margins to control the scalp bleeding. Subgaleal dissection was done to mobilize the posterior based flap. When sufficient mobilization of the flap was obtained, it was turned and used to cover the avulsed portion of the scalp. The flap was sutured to the wound margins with 2-0 Nylon sutures.

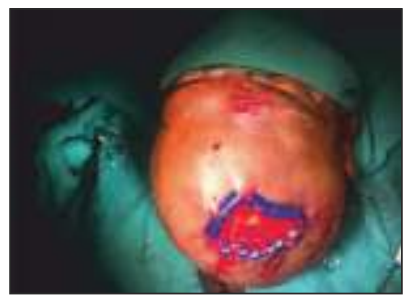

Figure 3 : Proper wound margins made

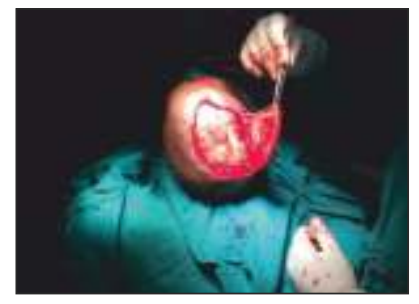

Figure 4 : M obilization of the flap and Raney clips placed

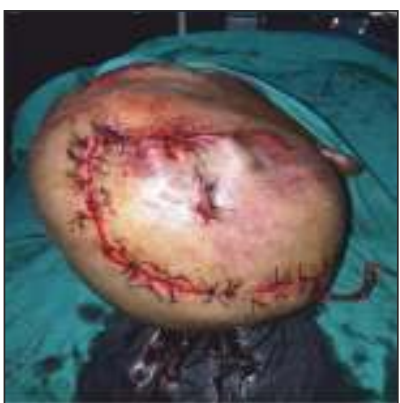

Figure 5 : After closure

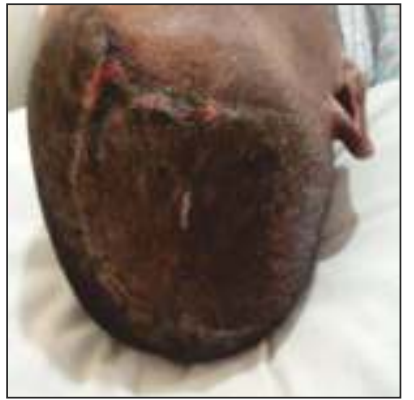

Figure 6 : Post operative Day 7

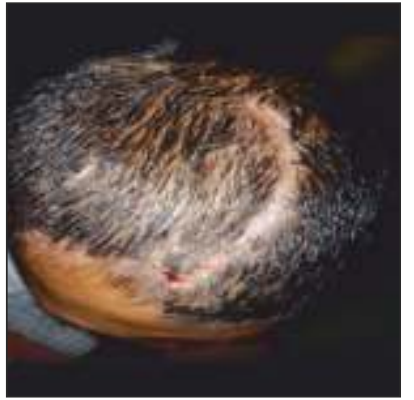

Figure 7 : Post operative day 15
Sutures were removed on post operative day 10. A satisfactory healing of the wound site was noted on the post operative day 15 , without any gaping. Hair growth was not observed during the defined follow up period, but is expected to be normal in subsequent visits.

\section{Discussion}

There are many options for repairing large scalp defects. The use of tissue expanders facilitates direct defect closure. This technique yields a larger amount of scalp tissue by 
placing an expanding device in the loose areolar tissue with good esthetic results. Expansion preferably should be carried out before removal of the lesion, and constitutes an excellent repair option for the reconstruction of defects following the resection of benign lesions. ${ }^{6}$ Healing by secondary intention is a possibility for repairing scalp defects, though this option is not applicable in the presence of large defects with a lack of pericranium. The use of free skin grafts for reconstruction requires an intact pericranium to supply vascularization to the repaired zone. In addition, careful hemostasis of the receptor bed is necessary, since the formation of hematomas would compromise graft success.

A basic principle in surgery is to first use the most simple technique available. In our case we presented a simple local flap procedure for repairing a large scalp defect that had an intact pericranium. ${ }^{1}$

When these flaps are applied to the scalp region, the edges of the wound must be carefully treated, taking care to avoid excessive tension at the extremities, since localized ischemia with involution of the hair follicles could result. Prior local anesthetic infiltration with epinephrine reduces bleeding at the wound edges, and facilitates dissection of the loose areolar plane. ${ }^{6}$ Electro cauterization is to be avoided, in order to reduce thermal damage to the hair follicles. The use of these flaps generally leaves "dog ears" at the end of surgery. When this happens, the temptation to resect them in the same surgical procedure is to be avoided, since they tend to disappear over time, and removal during surgery would increase tension upon the flap - thereby placing the extremities of the latter at risk. If

\section{References}

1. Frodel JLJr., Ahlstrom K. Reconstruction of complex scalp defects: the "Banana Peel" revisited. Arch Facial Plast Surg. 2004 Jan-Feb;6(1):5460.

2. Demir $Z^{1}$, Velidedeoglu $H$, Celebioglu S. V-Y-S plasty for scalp defects.PlastReconstr Surg. 2003 Sep 15;112(4):1054-8.

3. Mehrotra S, Nanda V, Shar RK. The islanded scal p flap: a better regional alternative to traditional flaps. PlastReconstr Surg. 2005 Dec;116(7):2039-40.

4. Newman $\mathrm{MI}^{1}$, Hanasono M M , Disa JJ, Cordeiro PG, M ehrara BJ. Scalp reconstruction: a 15-year experience. Ann Plast Surg. 2004 May;52(5):501-6; discussion 506. the esthetic deformity persists after a certain period of time, it can be corrected by means of a small operation under local anesthesia. ${ }^{6}$ In our patient, there was no significant dog ear formation post operatively, hence no revision procedure wasnecessary.

The anteroposterior midline of the scalp is a zone where the vascular territories of both sides of the cranium converge, forming a physiological barrier. It is advisable not to surpass this zone too much when preparing the flap. When the transverse posterior flap is fully located within the scalp region, as in our case, circulation is better and the risk of necrosis is reduced as a result. In the immediate postoperative period it is advisable to apply slight pressure to the flap, in order to avoid hematoma formation. Excessive pressure is to be avoided, however, since ischemia secondary to compression of the vascular pediclesmay result.

\section{Conclusion}

The present case describes a simple procedure for repairing a large scalp defect with intact pericranium, by means of a local Rotation Advancement flap. It is a viable option for treatment of such avulsed scalp defects or even other defects created post resection of scalp lesions. A significant esthetic factor is incorporated as the flap ensures a patent blood supply which in turn helps in hair growth in subsequent post operative period. The shortening of surgical time compared with other techniques, the simplicity of the surgical procedure, minimum morbidity and the satisfactory esthetic outcome make this an adequate option for repairing defects of this kind.

5. Michaelidis $\mathrm{IG}^{1}$, Stefanopoulos PK, Papadimitriou GA. The triple rotation scalp flap revisited: a case of reconstruction of cicatricial pressure alopecia. Int J Oral M axillofac Surg. 2006 Dec;35(12):1153-5. Epub 2006Jul 25.

6. Leedy JE', Janis JE, Rohrich RJ. Reconstruction of acquired scalp defects: an algorithmic approach. PlastReconstr Surg. 2005 Sep 15;116(4):54e-72e.

7. Tellioğlu $A T^{1}$, Cimen K, Açar HI, Karaeminogullar G, Tekdemir I. Scalp reconstruction with island hair-bearing flaps. PlastReconstr Surg. 2005 Apr 15;115(5):1366-71. 